\title{
Block-based tri-channel hybrid segmentation of images for foreground extraction
}

\author{
A SINDUJA* and A SURULIANDI \\ Department of Computer Science and Engineering, M S University, Abishekapatti 627012, India \\ e-mail: bloomingsindhuja@gmail.com; suruliandi@yahoo.com
}

MS received 22 October 2017; revised 14 February 2018; accepted 31 May 2018; published online 16 October 2018

\begin{abstract}
Image segmentation is crucial in image analysis, object representation, visualization and other image processing tasks. An image can be distinguished in terms of the foreground and the background. A new hybrid segmentation of images for foreground extraction is proposed, based on Interval Neutrosophic Set (INS) and Sparse Field Active Contour. In this method, an image is represented in three channels using a Gaussian filter bank and each channel is split into blocks to which the INS is applied. The resultant neutrosophic image for three channels undergoes isodata thresholding to obtain the tri-channel edge image, which is segmented using the Sparse Field Active Contour. The proposed method is evaluated by conducting three different experiments in natural image datasets like the Semantic Dataset100, Weizmann_Seg_DB_1obj, BSR and standard MATLAB test images. Finally, it is compared to other existing segmentation methods, which shows promising achievement in terms of their evaluation metrics like overlap-based metrics, pair-counting-based method and distance measures.
\end{abstract}

Keywords. Image segmentation; foreground extraction; hybrid segmentation; Interval Neutrosophic Set; Sparse Field Active Contour.

\section{Introduction}

The foreground is an integral part of the objective image and foreground segmentation is one of the major steps in many computer vision applications, like object recognition [1-3], object tracking [4], content-based image retrieval $[5,6]$, image editing [7], medical imaging $[3,8]$ and robot vision [9]. This segmentation process is found to be critical in image processing and pattern recognition. The criticality associated with this process can be overcome if segmentation is done with due diligence. In fact, there are no segmentation techniques applicable for images that can be said to work perfectly.

The acquired natural image can have a variety of intensities and be susceptible to conditions such as noise, orientation and pose. These characteristic make for the uncertain information in the image. The uncertainty is described using the Neutrosophic Set (NS), which is successfully used in image denoising, thresholding and segmentation. In this paper, block-based tri-channel hybrid segmentation is proposed using Interval Neutrosophic Set (INS) and Sparse Field Active Contour in which an image is converted into three channels and each channel into blocks for segmenting foreground from natural images. This proposed segmentation method allows managing the

*For correspondence uncertainty factor, overcoming complexities like noise and enhancing segmentation accuracy. Further, the generalization performance of the proposed technique is evaluated.

\subsection{Related works}

Many techniques for image segmentation have been developed by many researchers over the years. Some of the segmentation techniques are presented in this section.

1.1a General literature review on segmentation for background extraction: Dougherty presented the mathematical morphology [10] for segmenting the images of texture and particles. Fan et al [11] designed an automatic Seeded Region Growing (SRG) algorithm, along with a boundaryoriented parallel pixel labelling technique and an automatic seed selection method. Wang et al [12] introduced an algorithm of automatic foreground extraction for head shoulder images. This algorithm extracts the hard edge of foreground using an iterative graph cut method incorporated with an improved Gaussian Mixture Model. Stephanakis and Anastassopoulos proposed a novel algorithm based on adaptive thresholding of the global histogram of an image and it is applied for medical image segmentation [13]. Lee et al proposed an efficient foreground extraction algorithm [14], which can be applied for segmentation of the foreground from the images. Qi proposed the novel 
multilevel thresholds image segmentation method using maximum entropy and adaptive Particle Swarm Optimization (APSO) [15]. An automatic foreground extraction based on Difference of Gaussian (DoG) was proposed by Yuan et al [16]. Here, DoG was used to find the candidate keypoints of an input image, and foreground with the number of key points in each region was located using Normalized cut (Ncut). Qin et al [17] proposed a ViBebased method that employs simplified Gabor wavelets to calculate image edge information and it performs well in foreground-background segmentation and in colour shift situations caused by illumination or aperture adjustments. Yang et al [18] developed a new unsupervised algorithm to discover and segment out common objects from multiple images. Yang et al [19] proposed a method of spatially constrained energy function and Total Variation Flow Edge (TVFE) model for natural image segmentation. Pratondo et al [20] proposed a framework to integrate machine learning algorithms with region-based active contour models for medical image segmentation, which utilizes classification probability scores regularized using a nonlinear mapping. Akinlar and Topal [21] proposed a realtime greyscale edge and segment detector, Edge Drawing (GreyED), to detect edge segments in colour images and it is applied for natural images.

1.1b Literature review for NS: The NS was proposed by Smarandache [22] and it is useful for many segmentation algorithms in recent days. By means of NSs and Fuzzy C-Mean clustering (FCM), an improved segmentation approach was proposed by Anter et al [8]. A new concept of image thresholding is developed by Guo et al [23] based on neutrosophic similarity score for a variety of artificial and real images. Now the NS is defined as the set-theoretic operators on an instance of an NS and it is called as an INS $[24,25]$. As the INS is an extension of fuzzy set, it can solve certain problems that cannot be solved by fuzzy concepts. Hence, Zhang and Zhang [26] applied the INS to image processing and particularly in image segmentation. A new filtering method was presented by Mohan et al [27] to remove Rician noise from Magnetic Resonance Image based on the NS approach of Wiener filtering. Zhang et al [28] introduced a new concept of Neutrosophy $<$ Neut-A $>$, which represents the indeterminacy, and it is applied to three types of image segmentations like grey level images, breast ultrasound images and colour images. For noisy images in image processing, a novel image segmentation method based on NS and level set theory was proposed by Guo and Şengür [29]. Guo et al [30] applied the NS into image domain, and also defined some concepts and operators for image denoising.

1.1c Literature review for Sparse Field Active Contour: In image segmentation Active contour methods allow a contour to deform iteratively so that the images are partitioned into regions corresponding to the scene represented by an image [31]. Level set methods [32, 33] are often used for image segmentation because they are uncomplicated to implement however it is slow to compute. The drawback of level set method was overcome by Sparse Field Method (SFM) [34] and it was proposed by Whitaker which is used by Lankton to implement level set active contours efficiently [35]. Lucas, B.C., Kazhdan and Taylor presented a new method called Multi-Object Geodesic Active Contours (MOGAC) [36] which is the parallel sparse-field algorithm for image segmentation.

\subsection{Motivation}

In computer vision, image segmentation converts an image into a meaningful description that is relatively easy to analyse. However, it is hard to extract all meaningful objects from an image precisely and accurately because intensity, contrast, and blurring are certain features that affect the quality of segmentation. Several segmentation algorithms overcome these issues, though there still is a need to improve the quality of segmentation, given that the algorithms fail to deal with the noise present in the original image. Also, there is a need for easier segmentation along with a lack of redundancy. Motivated by this, an attempt is made to propose a block-based tri-channel hybrid segmentation method with the help of existing segmentation algorithms like the INS and the Sparse Field Active Contour. The proposed method is applied in natural images for foreground extraction. It can be justified from this that the edges are preserved by considering the image in three channels since the separation of channels using Gaussian filter has three different sigma value $(0.5,1$ and 1.5$)$ which retains the edges in one or the other channel. Also, the noise removal technique is mainly used for clear visibility of edges in the image by which the image gets blurred - with the white pixel at the center and the dark pixels near the edges by using Gaussian filter for channel separation. The blurring of image can clearly say that the noise is getting suppressed. Basically, the block separation will enhance the edge but in rectangular block separation, the differentiation of the boundary between the background and the foreground is much more precise and the issue of redundancy is overcome as well. In general, an image has uniform objects and background, and blurry edges are gradually changing from objects to background. Hence, in the INS, intermediate set is identified by means of homogeneity value since this value is larger for objects and background than that of edges. Here the canny edge detector is used to improve the homogeneity value dealt with the issue of blurry edge, vital to image segmentation.

\subsection{Outline of the proposed work}

This section briefly explains the overall process of the segmentation method.

The outline of the proposed method is described in figure 1. Here, the block-based tri-channel hybrid 


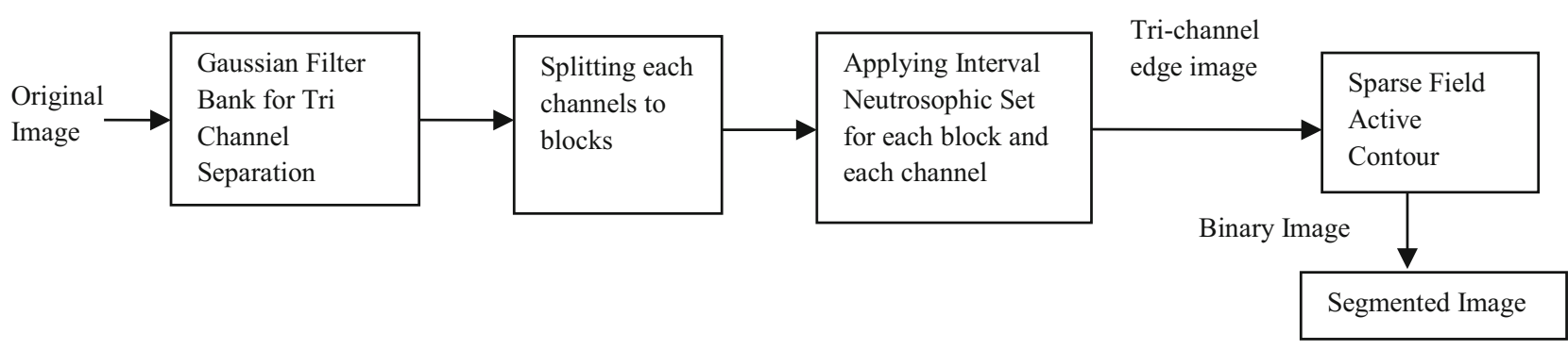

Figure 1. Outline of the proposed segmentation method.

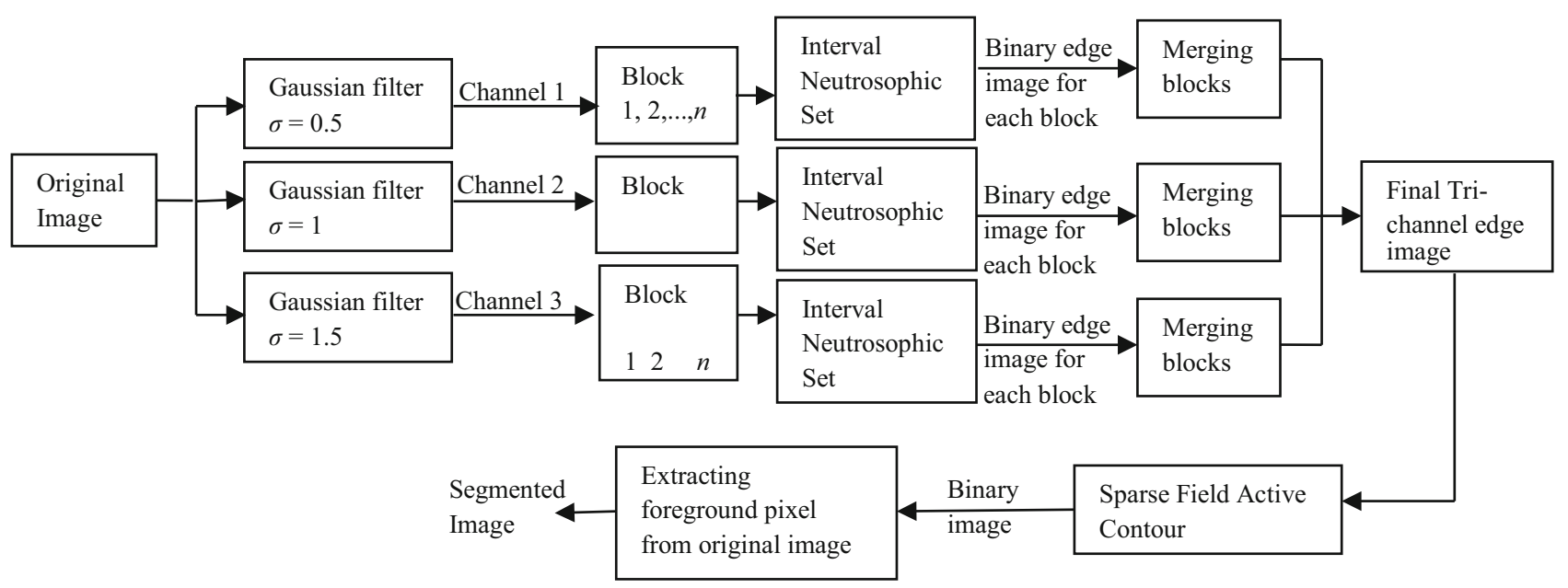

Figure 2. Block-based tri-channel hybrid segmentation method.

segmentation method is proposed. Firstly, a natural image is given as an input, to which the Gaussian filter bank is applied for converting the single greyscale image into three channel images. Thereafter, the block-based INS is applied to the three channels. Finally, the binary image is obtained by applying the Sparse Field Active Contour in the trichannel edge image. This binary image is compared with the original image to obtain the foreground object.

\subsection{Organization of the paper}

This paper is organized as follows. Section 2 explains the proposed segmentation method along with the existing segmentation algorithm. In section 3, the experimental results and in section 4, performance analysis are explored. Section 5 concludes the paper.

\section{Block-based tri-channel hybrid segmentation method}

The segmented image is obtained in two modules. In the first module, a tri-channel edge image is obtained. In the second module, the Sparse Field Active Contour is applied to the tri-channel edge image and the final segmented results are obtained.

In figure 2, the overall process for the first module in the segmentation method is composed of four steps: (1) The original image is converted into three multiresolution channels using the Gaussian filter bank, (2) the obtained images are divided into $128 \times 8$ overlapping blocks, (3) the INS is applied to each block from which a binarized image is obtained containing binary edge value and (4) the resultant overlapping blocks are merged into a single binary image. Steps 2-4 are applied to all the three multiresolution images. Finally, the three resultant images are converted into a tri-channel edge image. In the second module, Sparse Field Active Contour is applied to the tri-channel edge image to extract foreground pixels from the original image.

\subsection{Separation of tri-channels and blocks}

To extend the standard NS, here three channel images from the original image are obtained using the Gaussian filter bank [37], described in Eq. (1):

$$
G(n, m, \sigma)=\frac{1}{2 \pi \sigma^{2}} \mathrm{e}^{-\left(n^{2}+m^{2}\right) / 2 \sigma^{2}} .
$$




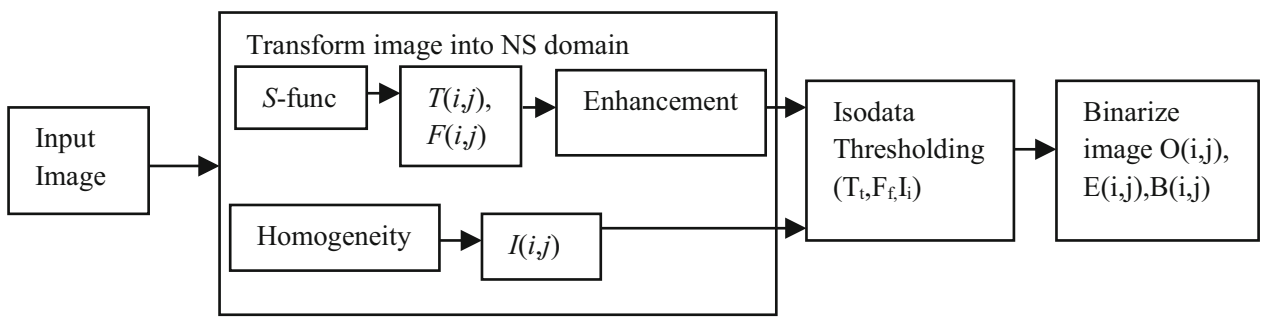

Figure 3. Flowchart for Interval Neutrosophic Set.

From Eq. (1), the Gaussian kernel with the scale and kernel mask is obtained. The three multiresolution images of the given natural image are calculated as follows:

$$
L(\sigma)=G(n, m, \sigma) * I(n, m)
$$

where $\sigma$ represents different scales and * indicates the convolution operator. In this work, the impact of different combinations of $\sigma 1, \sigma 2$ and $\sigma 3$ with various convolution masks is analysed and the optimum values of $0.5,1$ and 1.5 with a convolution mask of size 7,7 are used.

The three channels are divided into overlapping blocks of size $128 \times 8$, and the INS is applied to each block to obtain the binary image.

\subsection{INS}

The INS is an instance of an NS that can be used in realtime scientific and engineering applications.

Definition 1 (INS) Let $\mathrm{X}$ be a space of points (objects) with a generic element in $\mathrm{X}$ denoted by $\mathrm{x}$. An INS, N, in X is characterized by the truth-membership function $T_{\mathrm{N}}$, indeterminacy-membership function $I_{\mathrm{N}}$ and falsity-membership function $F_{\mathrm{N}}$. For each point $\mathrm{x}$ in $\mathrm{X}, T_{\mathrm{N}}(\mathrm{x}), I_{\mathrm{N}}(\mathrm{x})$, $F_{\mathrm{N}}(\mathrm{x}) \subseteq[0,1][26]$.

Definition 2 (Interval Neutrosophic Image) Let U be a universe of discourse, and an INS, N, be included in U [26]. An interval neutrosophic image, $\mathrm{P}_{\text {INS }}$, is characterized by three subsets $T, I$ and $F$, and $T, I, F \subseteq[0,1]$. According to this definition, the element $\mathrm{P}(T, I, F)$ belongs to $\mathrm{N}$ in the following way: it is $t \%$ true in the set, $i \%$ indeterminate in the set and $f \%$ false, where $t$ varies in T, $i$ varies in I and $\mathrm{f}$ varies in $\mathrm{F}$. A pixel, $P$, in the interval neutrosophic image is described as follows:

$$
P(i, j)=\{T(i, j), I(i, j), F(i, j)\}
$$

where $T(i, j), I(i, j)$ and $F(i, j)$ are the probabilities that belong to the white set, indeterminate set and non-white set, respectively [8].

Figure 3 explains the INS. In this work, the input image is mapped to $T$ and $F$ by the $S$-function and to $I$ by the homogeneity value. The interval neutrosophic image mapping $[28,38]$ is described as follows:

$$
T(i, j)= \begin{cases}1 & 0 \leq \operatorname{Img}_{i, j} \leq a \\ 1-\frac{\left(\operatorname{Img}_{i, j}-a\right)^{2}}{(c-a)(b-a)} & a<\operatorname{Img}_{i, j} \leq b \\ \frac{\left(\operatorname{Img}_{i, j}-a\right)^{2}}{(c-b)(c-a)} & b<\operatorname{Img}_{i, j} \leq c \\ 0 & \operatorname{Img}_{i, j} \geq c\end{cases}
$$

where $\operatorname{Img}_{i, j}$ is the intensity of pixel $P(i, j)$ and $i, j$ is the position. Variables $a, b$ and $c$ are the parameters that determine the shape of the $S$-function. A histogram-based method is used to calculate the variables $a, b$ and $c$ [39].

$$
\begin{gathered}
I(i, j)=1-H(i, j), \\
H(i, j)=1-\frac{\mathrm{SD}(i, j)}{\mathrm{SD}_{\max }} \times \frac{\mathrm{eg}(i, j)}{\mathrm{eg}_{\max }} .
\end{gathered}
$$

Homogeneity, defined by standard deviation, describes the contrast within a local region and the discontinuity of the intensity that is about to change in grey levels.

The discontinuity of the intensity, $P$, can be described by the edge value and there is hence a need for an edge operator. Here, the canny operator is used to find discontinuities.

$$
\operatorname{eg}(i, j)=\sqrt{G_{i}^{2}+G_{j}^{2}}
$$

The standard deviation of the pixel, $P$, is computed for a window size $w \times w$ centred at $(i, j)$ :

$$
\begin{gathered}
\mathrm{SD}(i, j)=\sqrt{\frac{\sum_{x=i-(w-1) / 2}^{i+(w-1) / 2} \sum_{\mathrm{y}=j-(w-1) / 2}^{\mathrm{j}+(w-1) / 2}\left(\operatorname{Img}_{x, y}-\mu_{i, j}\right)^{2}}{w^{2}}} \\
\mu_{i, j}=\sum_{x=i-(w-1) / 2}^{i+(w-1) / 2} \sum_{y=j-(w-1) / 2}^{j+(w-1) / 2} \frac{\operatorname{Img}_{x, y}}{w^{2}}
\end{gathered}
$$

$\mu_{i, j}$ is the mean value of the intensity within the window $w \times w$. 


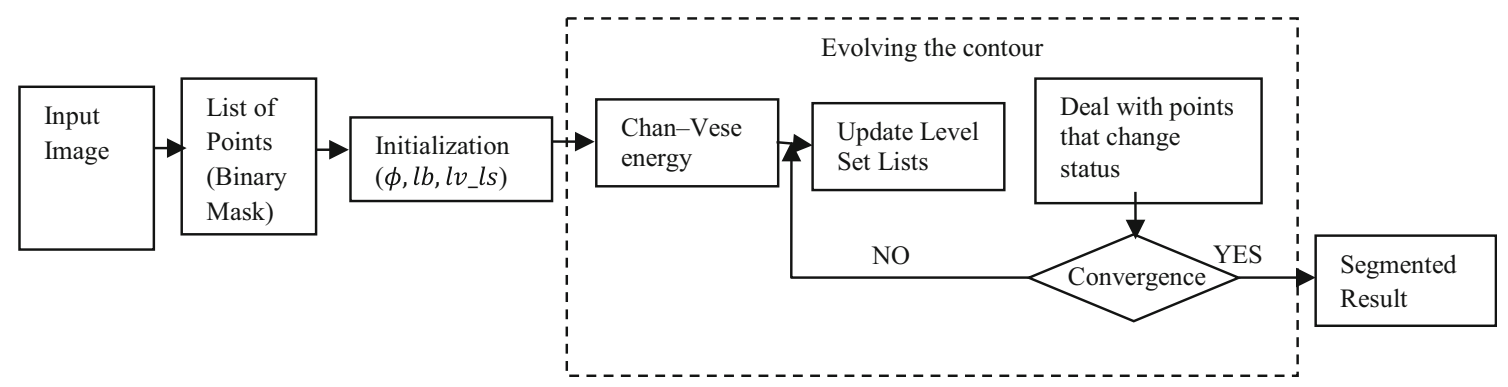

Figure 4. Sparse Field Active Contour.

2.2a Enhancement of the INS domain: The INS domain image is enhanced using intensification transformation to make segmentation easier and more effective [38].

$$
\begin{gathered}
T_{\mathrm{E}}(i, j)=2 T_{\mathrm{E}}^{2}(i, j), \\
T_{\mathrm{E}}(i, j)=1-2\left(1-T_{\mathrm{E}}(i, j)\right)^{2}, \\
F_{\mathrm{E}}(i, j)=1-T_{\mathrm{E}}(i, j) .
\end{gathered}
$$

$2.2 \mathrm{~b}$ Isodata threshold: The threshold values for $T_{\mathrm{E}}, F_{\mathrm{E}}$ and $I_{\mathrm{i}}$ are needed for converting the INS to the binary image; here, isodata thresholding is used for finding $T_{\mathrm{t}}, F_{\mathrm{f}}$ and $I_{\mathrm{i}}$, respectively.

1. For calculating the threshold value for white set $\left(T_{\mathrm{f}}\right)$ the INS image is converted to a histogram.

2. The mean intensity, $T$, of the image ( $T=$ mean of image) from the histogram is computed.

3. The Mean Above $T$ (MAT) and the Mean Below $T$ (MBT) are computed using $T$ from step 2.

4. The average of these two sample means gives the new threshold value. The process is repeated, based on the new threshold value, until the threshold value does not change any more.

5. These four steps are repeated for calculating the threshold value for remaining two sets like indeterminate set $\left(I_{\mathrm{i}}\right)$ and non-white set $\left(F_{\mathrm{E})}\right.$.

The INS image is divided into three parts: $T(i, j)$ describes the degree of being an object $(O)$ pixel, $I(i, j)$ is the degree of being an edge $(E)$ pixel and $F(i, j)$ is the degree of being a background $(B)$ pixel for pixel $P(i, j)$. The three parts are defined as follows [38]:

$$
\begin{aligned}
& O(i, j)= \begin{cases}1 & T(i, j) \geq T_{\mathrm{t}}, I(i, j)<I_{\mathrm{i}}, \\
0 & \text { otherwise }\end{cases} \\
& E(i, j)= \begin{cases}1 & T(i, j)<T_{\mathrm{t}} \vee F(i, j)<F_{\mathrm{f}}, I(i, j) \geq I_{\mathrm{i}} \\
0 & \text { otherwise }\end{cases} \\
& B(i, j)= \begin{cases}1 & F(i, j) \geq F_{\mathrm{f}}, I(i, j)<I_{\mathrm{i}} \\
0 & \text { otherwise }\end{cases}
\end{aligned}
$$

From the obtained results, the objects and background are mapped to 1 , and the edges are mapped to 0 in the binary image. The mapping functions are as follows:

$$
\operatorname{binary}(i, j)= \begin{cases}1 & O(i, j) \vee B(i, j) \vee \overline{E(i, j)}=\text { true } \\ 0 & \text { others }\end{cases}
$$

\subsection{Sparse field active contour}

Level set functions $[32,33]$ are used to segment the image into different regions. The interfaces separating those regions are defined as the zeros of the level set function. The SFM [35] uses lists that contain points in a zero level set as well as points near the zero level set. The neighbourhood of the zero level set is computed each time using the approximation to the distance function, which gives this type of computation the flexibility needed. It is feasible to use a linked list to keep track of the list of points available. This process involves two steps, as shown in figure 4.

Figure 4 depicts the Sparse Field Active Contour image segmentation method and the steps for obtaining the segmented image using Sparse Field Active Contour are as follows.

1. The image obtained from section 2.3 is considered as input image for this method.

2. A binary mask is created using the values 0 and 1 , which denote background pixels and foreground pixels, respectively, and is fed as input image for the initialization stage.

3. Three arrays are returned from the initialization stage. The first array is the label map (lb), which is used to record the status of each pixel, and searches for which list a pixel belongs to. The second is the $\phi$ (phi) array. This is an arbitrary function that is maintained as long as the zero level set gives us the contour and the third array is the five-level lists (lv_ls), which hold the $x, y$ and $z$ locations of pixels in the image [35].

4. After the initialization process, energy is calculated for the active contour. Here, Chan-Vese energy $(F)$ is considered.

5. Now the $\phi$ value is updated along the zero-level set and the remaining four-level lists of the adjacent points. For this process, five additional lists are used for holding points temporarily, which change the status of the points. 


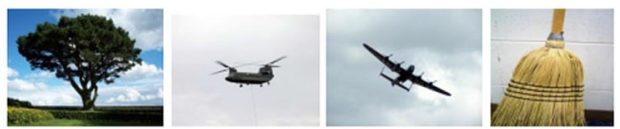

(a) Weizmann_Seg_DB_1obj

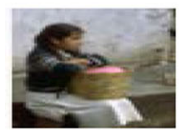

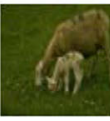

(c) BSR

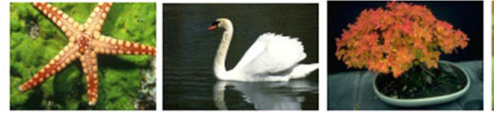

(b) Semantic dataset100

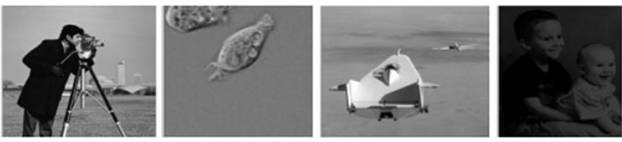

(d) Standard MATLAB test image

Figure 5. Sample natural images.

6. After all the points in the entire level lists have been iterated, the points that have changed their status during the iteration have to be processed.

7. Steps 4-6 are repeated until convergence is reached and in step 4 , the energy value, $F$, can be computed again, based on the contour's new position.

8. Finally, the segmented result is obtained from the Sparse Field Active Contour.

\subsection{Procedure for block-based tri-channel hybrid segmentation method:}

1. Natural image is given as the input of size $256 \times 256$.

2. For the given image I, Gaussian filter is applied with three different sigma values to convert the image into three channels $\mathrm{C}(:,:, 3)$ of size $256 \times 256 \times 3$ (in section 2.1).

3. The channel $\mathrm{I}_{1}$ is divided into $N$ number of overlapping blocks of size $128 \times 8\left(\mathrm{I}_{11}, \mathrm{I}_{12}, \ldots, \mathrm{I}_{1 \mathrm{~N}}\right)$ using sliding window concept.

4. The INS (in section 2.2) is then applied to each image block $\left(\mathrm{I}_{11}, \mathrm{I}_{12}, \ldots, \mathrm{I}_{1 \mathrm{~N}}\right)$ and the binary edge images $\mathrm{B}_{11}$, $\mathrm{B}_{12}, \ldots \mathrm{B}_{1 \mathrm{~N}}$ are obtained for each block $\left(\mathrm{I}_{11}, \mathrm{I}_{12}, \ldots\right.$, $\mathrm{I}_{1 \mathrm{~N}}$ ).

5. All these blocks are merged to obtain the single binary edge $\mathrm{B}_{1}$ image for channel $\mathrm{I}_{1}$.

6. Steps $3-5$ are repeated for remaining two channels $I_{2}$ and $\mathrm{I}_{3}$.

7. Finally, three binary edge images $\left(\mathrm{B}_{1}, \mathrm{~B}_{2}\right.$ and $\left.\mathrm{B}_{3}\right)$ are obtained for all the three channels.

8. Binary edge images $B_{1}, B_{2}, B_{3}$ are combined to obtain the new tri-channel edge image $\mathrm{N}(:,:, 3)$.

9. Tri-channel image $\mathrm{N}$ is given as the input for Sparse Field Active Contour (in section 2.3), from which the segmented binary image $S$ is obtained by differentiating the object ' 0 ' and the background ' 1 '.

10. Comparing segmented binary image $S$ with tri-channel image $\mathrm{C}$ to extract the foreground portion in the image.

\section{Experimental results}

In this section, the performance of the proposed segmentation method is analysed.

\subsection{The dataset}

A dataset with natural images is collected. Four different datasets like the Semantic Dataset100, BSR, Weizmann_Seg_DB_1obj and standard MATLAB test image datasets are considered, from which around 50, 35, 40 and 21 images are selected, respectively, for four different experiments.

Sample images from all the datasets are shown in figure 5 . The image resolution is $256 \times 256$. The images are converted to three channels and each channel is divided into overlapping rectangular blocks, since the block size must include enough local information.

\subsection{Experimental output}

Experiments are conducted to evaluate the proposed segmentation approach, and analysed with the existing segmentation algorithm to evaluate the best. The output obtained from the segmentation approach is presented in table 1.

Table 1 shows that the binary edge image is obtained by the block-based tri-channel INS, and from the edge image, the foreground pixels are segmented using the Sparse Field Active Contour. The obtained results demonstrate that the proposed method for edge detection from a natural image is accurate and provides good segmentation results.

\section{Performance analysis}

In this section, the first and second experiments are analysed using the true positive rate (TPR), true negative rate (TNR), false positive rate (FPR), false negative rate and 
Table 1. Segmentation results for sample images.

\begin{tabular}{|c|c|c|c|c|}
\hline Dataset name & Image name/no. & Input samples & INS results & $\begin{array}{c}\text { Sparse Field Active } \\
\text { Contour results }\end{array}$ \\
\hline \multirow{4}{*}{$\begin{array}{c}\text { Weizmann_Seg_ } \\
\text { DB_1obj }\end{array}$} & 100_0109 & & & \\
\hline & b2chopper008 & & & \\
\hline & $\begin{array}{c}\text { bbmf_lancaster_ } \\
\text { july_06 }\end{array}$ & & & \\
\hline & broom 07 & & & \\
\hline \multirow{4}{*}{ Semantic dataset 100} & 12003 & & & \\
\hline & 8068 & & & \\
\hline & 353013 & & & \\
\hline & 189011 & & & \\
\hline \multirow{4}{*}{ BSR } & 23025 & & & \\
\hline & 24063 & & & \\
\hline & 49024 & & & \\
\hline & 207056 & & & \\
\hline \multirow{4}{*}{$\begin{array}{l}\text { Standard MATLAB } \\
\text { test image }\end{array}$} & Cameraman & & & \\
\hline & Cell & & & \\
\hline & Liftingbody & & & \\
\hline & Kids & & & \\
\hline
\end{tabular}

accuracy. The third experiment is evaluated using the peak signal-to-noise ratio (PSNR) and the mean square error (MSE). The fourth experiment is evaluated using different metrics like overlap-based metrics, pair-counting-based metrics and distance measures.

\subsection{Performance metrics}

The segmentation quality is assessed by performance metrics chosen on the properties of each measure and their use. Four cardinalities, true positive (TP), false positive 
(FP), true negative (TN) and false negative (FN), are obtained from the two crisp segmentations. From these cardinalities, a few definitions for the performance measures are given as follows.

4.1a TPR: TPR [40] or sensitivity relates to the test's ability to measure the proportion of the foreground that is correctly segmented as such:

$$
\mathrm{TPR} \text { or sensitivity }=\frac{\mathrm{TP}}{\mathrm{TP}+\mathrm{FN}} \text {. }
$$

4.1b TNR: TNR [40] or specificity relates to the test's ability to measure the proportion of the background that is correctly segmented as such:

$$
\mathrm{TNR} \text { or specificity }=\frac{\mathrm{TN}}{\mathrm{TN}+\mathrm{FP}} .
$$

The values of the TPR and the TNR should be high, as these metrics provide the proportion of the correctly segmented foreground and background.

4.1c FPR: FPR [40] or fallout usually refers to the probability of falsely rejecting the foreground in the image:

$$
\text { FPR or fallout }=\frac{\mathrm{FP}}{\mathrm{FP}+\mathrm{TN}} .
$$

4.1d FNR: FNR [40] is the proportion of the background that is falsely interpreted as the true foreground in the image:

$$
\mathrm{FNR}=\frac{\mathrm{FN}}{\mathrm{TP}+\mathrm{FN}}
$$

The values of the FPR and the FNR should be low, as these metrics provide the proportion of the falsely segmented foreground and background.

4.1e Positive predictive value $(P P V)$ : The positive predictive value (PPV) [40] is defined as the ratio of the truly segmented foreground to the all correctly and falsely segmented foreground:

$$
\text { precision or } \mathrm{PPV}=\frac{\mathrm{TP}}{\mathrm{TP}+\mathrm{FP}} \text {. }
$$

4.1f Accuracy: Accuracy [40] refers to the closeness of a segmented image to the ground truth image:

$$
\text { accuracy }=\frac{\mathrm{TP}+\mathrm{TN}}{\mathrm{TP}+\mathrm{FP}+\mathrm{TN}+\mathrm{FN}} .
$$

4.1g $F_{1}$-measure: The $F_{1}$ score [40] (also $F$-score or $F$ measure) uses both precision and recall for the score:

$$
\mathrm{F}_{1} \text {-measure }=\frac{2 \mathrm{PPV} . \mathrm{TPR}}{\mathrm{PPV}+\mathrm{TPR}}
$$

The PPV, accuracy and $F_{1}$-measure values should be high for better segmentation results.

4.1h DICE: DICE [40] is a statistic used to compare the resemblance between segmented and ground truth images:

$$
\mathrm{DICE}=\frac{2 \mathrm{TP}}{2 \mathrm{TP}+\mathrm{FP}+\mathrm{FN}}
$$

4.1i Jaccard index (JAC): The Jaccard index (JAC) [7] is a statistic used to compare the resemblance and diversity between segmented and ground truth images:

$$
\mathrm{JAC}=\frac{\mathrm{TP}}{\mathrm{TP}+\mathrm{FP}+\mathrm{FN}}
$$

4.1j Rand index: The Rand index (RI) [40] measures the similarity between the test and ground truth segmentations. It is given by the sum of the number of pairs of pixels that have the same label in the test and ground truth segmentations and those that have different labels in both

$$
\mathrm{RI}=\frac{a+b}{a+b+c+d}
$$

4.1k Adjusted Rand index (ARI): The adjusted Rand index (ARI) [40] is the variation of the Rand index from the estimated value if the marginal distributions of the contingency table were independent:

$$
\begin{aligned}
& \text { adjusted Rand index }(\mathrm{ARI}) \\
& =\frac{2(a d-b c)}{c^{2}+b^{2}+2 a d+(a+d)(c+b)}
\end{aligned}
$$

where $\quad a=\frac{1}{2}[\mathrm{TP}(\mathrm{TP}-1)+\mathrm{FP}(\mathrm{FP}-1)+\mathrm{TN}(\mathrm{TN}-1)+$ $\mathrm{FN}(\mathrm{FN}-1)], \quad b=\frac{1}{2}\left[(\mathrm{TP}+\mathrm{FN})^{2}+(\mathrm{TN}+\mathrm{FP})^{2}-\left(\mathrm{TP}^{2}+\right.\right.$ $\left.\left.\mathrm{TN}^{2}+\mathrm{FP}^{2}+\mathrm{FN}^{2}\right)\right], \quad c=\frac{1}{2}\left[(\mathrm{TP}+\mathrm{FP})^{2}+(\mathrm{TN}+\mathrm{FN})^{2}-\right.$ $\left.\left(\mathrm{TP}^{2}+\mathrm{TN}^{2}+\mathrm{FP}^{2}+\mathrm{FN}^{2}\right)\right], d=\frac{n(n-1)}{2-(a+b+c)}$ and $n=\mathrm{TP}+$ $\mathrm{FP}+\mathrm{TN}+\mathrm{FN}$.

The values of the DICE, JAC, RI and ARI must be high, since they are obtained by comparing the similarity between the segmented and ground truth images.

4.11 Structural SIMilarity (SSIM) index: The Structural SIMilarity (SSIM) [41] index is used to measure the similarity between two images. The calculation is done between two segmentation methods of size $N \times N$ using the relation

$$
\operatorname{SSIM}(X, Y)=\frac{(2 \mu \mathrm{X} \mu \mathrm{Y}+C 1)(2 \sigma x y+C 2)}{\left(\mu \mathrm{X}^{2}+\mu \mathrm{Y}^{2}+C_{1}\right)\left(\sigma \mathrm{X}^{2}+\sigma \mathrm{Y}^{2}+C_{2}\right)}
$$

where $\mu \mathrm{X}$ is the average of $X, \mu \mathrm{Y}$ is the average of $Y$, and $\sigma \mathrm{X}^{2}$ and $\sigma \mathrm{Y}^{2}$ are the variance of $X$ and $Y$, respectively. $\sigma \mathrm{XY}$ is the covariance of $X$ and $Y . C_{1}$ and $C_{2}$ are the variables that stabilize the weak denominator. The SSIM value is 1 if there are two identical sets of images.

$4.1 \mathrm{~m}$ Variation of Information (VOI): The variation of information (VOI) [42] metric defines the amount of randomness in one segmentation which cannot be explained by the other segmentation: 
Table 2. Segmentation evaluation for non-overlapping blocks by varying window size.

\begin{tabular}{|c|c|c|c|c|c|c|}
\hline Block size & Dataset & TPR & TNR & FPR & FNR & Accuracy \\
\hline \multirow[t]{5}{*}{$128 \times 8$} & Semantic dataset 100 & 0.69 & 0.77 & 0.08 & 0.30 & 0.85 \\
\hline & BSR & 0.55 & 0.76 & 0.09 & 0.25 & 0.82 \\
\hline & Weizmann_Seg_DB_1obj & 0.65 & 0.78 & 0.07 & 0.34 & 0.85 \\
\hline & Standard test image & 0.56 & 0.78 & 0.07 & 0.43 & 0.81 \\
\hline & Average & 0.61 & 0.77 & 0.07 & 0.43 & 0.83 \\
\hline \multirow[t]{5}{*}{$128 \times 16$} & Semantic dataset 100 & 0.55 & 0.69 & 0.15 & 0.50 & 0.75 \\
\hline & BSR & 0.56 & 0.78 & 0.07 & 0.43 & 0.81 \\
\hline & Weizmann_Seg_DB_1obj & 0.43 & 0.67 & 0.13 & 0.57 & 0.70 \\
\hline & Standard test image & 0.45 & 0.70 & 0.15 & 0.54 & 0.73 \\
\hline & Average & 0.49 & 0.71 & 0.12 & 0.51 & 0.74 \\
\hline \multirow[t]{5}{*}{$128 \times 32$} & Semantic dataset 100 & 0.49 & 0.68 & 0.17 & 0.50 & 0.72 \\
\hline & BSR & 0.49 & 0.65 & 0.14 & 0.50 & 0.68 \\
\hline & Weizmann_Seg_DB_1obj & 0.55 & 0.68 & 0.19 & 0.45 & 0.70 \\
\hline & Standard test image & 0.40 & 0.7 & 0.15 & 0.60 & 0.73 \\
\hline & Average & 0.48 & 0.67 & 0.16 & 0.51 & 0.70 \\
\hline \multirow[t]{5}{*}{$128 \times 64$} & Semantic dataset100 & 0.40 & 0.58 & 0.16 & 0.60 & 0.68 \\
\hline & BSR & 0.38 & 0.66 & 0.14 & 0.45 & 0.72 \\
\hline & Weizmann_Seg_DB_1obj & 0.43 & 0.56 & 0.25 & 0.56 & 0.65 \\
\hline & Standard test image & 0.39 & 0.71 & 0.14 & 0.60 & 0.72 \\
\hline & Average & 0.40 & 0.62 & 0.17 & 0.55 & 0.69 \\
\hline
\end{tabular}

Table 3. Segmentation evaluation for overlapping blocks by varying window size.

\begin{tabular}{|c|c|c|c|c|c|c|}
\hline Block size & Images & TPR & TNR & FPR & FNR & Accuracy \\
\hline \multirow[t]{5}{*}{$128 \times 8$} & Semantic dataset100 & 0.62 & 0.92 & 0.07 & 0.35 & 0.84 \\
\hline & BSR & 0.65 & 0.92 & 0.07 & 0.34 & 0.84 \\
\hline & Weizmann_Seg_DB_1obj & 0.68 & 0.92 & 0.07 & 0.30 & 0.86 \\
\hline & Standard test image & 0.61 & 0.92 & 0.07 & 0.38 & 0.83 \\
\hline & Average & 0.64 & 0.92 & 0.07 & 0.34 & 0.84 \\
\hline \multirow[t]{5}{*}{$128 \times 16$} & Semantic dataset 100 & 0.50 & 0.82 & 0.17 & 0.50 & 0.75 \\
\hline & BSR & 0.51 & 0.83 & 0.16 & 0.48 & 0.73 \\
\hline & Weizmann_Seg_DB_1obj & 0.49 & 0.82 & 0.17 & 0.50 & 0.72 \\
\hline & Standard test image & 0.50 & 0.84 & 0.15 & 0.49 & 0.71 \\
\hline & Average & 0.50 & 0.82 & 0.16 & 0.49 & 0.72 \\
\hline \multirow[t]{5}{*}{$128 \times 32$} & Semantic dataset 100 & 0.57 & 0.80 & 0.19 & 0.43 & 0.73 \\
\hline & BSR & 0.58 & 0.87 & 0.12 & 0.42 & 0.77 \\
\hline & Weizmann_Seg_DB_1obj & 0.38 & 0.80 & 0.16 & 0.62 & 0.63 \\
\hline & Standard test image & 0.40 & 0.83 & 0.35 & 0.60 & 0.69 \\
\hline & Average & 0.48 & 0.82 & 0.20 & 0.51 & 0.70 \\
\hline \multirow[t]{5}{*}{$128 \times 64$} & Semantic dataset 100 & 0.43 & 0.85 & 0.14 & 0.55 & 0.72 \\
\hline & BSR & 0.39 & 0.83 & 0.19 & 0.61 & 0.70 \\
\hline & Weizmann_Seg_DB_1obj & 0.48 & 0.76 & 0.23 & 0.51 & 0.70 \\
\hline & Standard test image & 0.50 & 0.74 & 0.25 & 0.49 & 0.68 \\
\hline & Average & 0.45 & 0.79 & 0.20 & 0.54 & 0.70 \\
\hline
\end{tabular}

$$
\operatorname{VOI}(X, Y)=H(X)+H(Y)-2 I(X, Y)
$$

where $H(X)$ and $H(Y)$ are entropies associated with $X$ and $Y$, respectively, and $I(X, Y)$ is the mutual information between $X$ and $Y$.

4.1n Global Consistency Error (GCE): The global consistency error (GCE) [42] measures the consistency of an image with respect to another image, related to each other, even if they are on different scales:

$$
\begin{aligned}
& \text { global consistency error (GCE) } \\
& \qquad=\frac{1}{n} \min \left\{\sum_{k} E_{k}(S, M), \sum_{k} E_{k}(M, S)\right\}
\end{aligned}
$$


Let $\mathrm{S}$ and $\mathrm{M}$ be two segmentations. For a given pixel, $E_{k}$, consider the segments that contain $E_{k}$ in $\mathrm{S}$ and M. VOI and GCE have a value of zero if the segment is a proper subset of the other segment.

4.1o PSNR: PSNR [43] represents the region homogeneity of the final partitioning. The higher the value of the PSNR, the better the segmentation of the image:

$$
\mathrm{PSNR}=10 \log _{10}\left(\frac{255^{2}}{\mathrm{MSE}}\right) .
$$

The MSE [43] of the segmented image is computed as

$$
\mathrm{MSE}=\frac{1}{M N} \sum \sum|S(i, j), F(i, j)|
$$

where $S$ is the segmented image and $F$ is the source image with a pixel size of $M \times N$.

\subsection{Performance evaluation by varying block category and block size}

Before converting the image into tri-channels, the best block size and block category should be analysed for blockbased segmentation. Here, the images are downsampled to block sizes $128 \times 8,128 \times 16,128 \times 32$ and $128 \times 64$ for both categories. Most objects in natural images are found to be at the centre and also there is the need for pursuing exact boundaries of the object; therefore, the rectangular blocks are considered. Tables 2 and 3 show the TPR, TNR, FPR, FNR and accuracy by varying the block size for overlapping and non-overlapping block categories, respectively.

From tables 2 and 3, it is noted that the block size of $128 \times 64$ encloses a neighbouring region of the object. At the same time, however, for a larger block size there is every probability of having multiple objects in a single block, which is the least conducive for segmentation. On the other hand, the block size of $128 \times 8$ produces promising results for segmentation, since there is a higher probability that a smaller block size contains a single object: consequently, the information about the object is much more descriptive. It is also noted that in overlapping block-based segmentation, the images are split into blocks using the sliding window concept and there is the possibility of reconsidering important pixel values in each block. This is precisely why this segmentation method provides better results than non-overlapping block-based segmentation.

\subsection{Result evaluation for the proposed approach by varying the convolution mask and sigma value}

The block-based tri-channel INS with Sparse Field Active Contour was analysed for the block size of $128 \times 8$ as it

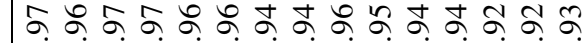

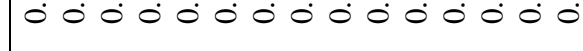

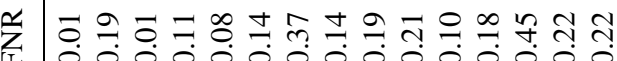

先

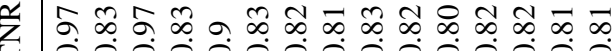
ப்

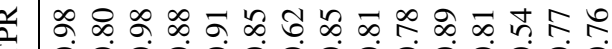

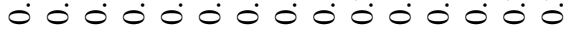

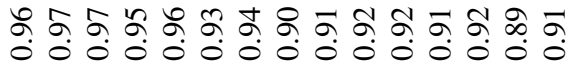
\&

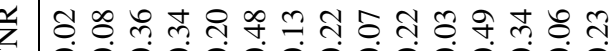
000000000000000

藏

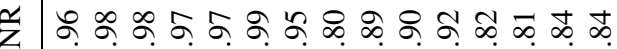

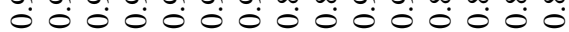

츨

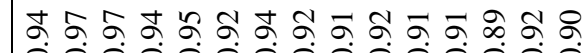
00000000000000

艺 瓷

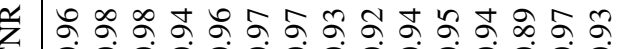
००००0:00000000000

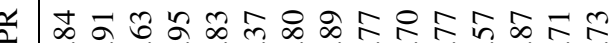

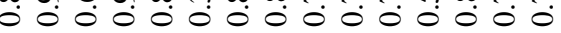


Table 5. Result evaluation of de-noising capability for tri-channel block-based INS.

\begin{tabular}{lccrr}
\hline \multirow{2}{*}{ Datasets } & \multicolumn{2}{c}{ Without tri-channel } & \multicolumn{2}{c}{ With tri-channel } \\
\cline { 2 - 3 } \cline { 3 - 5 } PSNR & MSE & 30.4596 & 58.2561 & 0.0981 \\
\hline Semantic dataset100 & 33.2658 & 37.6152 & 58.7299 & 0.0878 \\
BSR & 32.3369 & 49.0276 & 58.8685 & 0.0856 \\
Weizmann_Seg_DB_1obj & 31.2965 & 38.9612 & 58.2055 & 0.0987 \\
Standard test image & 32.2693 & MSE \\
\hline
\end{tabular}

gave good results in the previous experiment. Here, the image is converted into a tri-channel using the Gaussian filter bank. The impact of different combinations of $\sigma 1, \sigma 2$ and $\sigma 3$ with various convolution masks is analysed to find the optimum values of sigma and the convolution mask size, shown in table 4 . Here, the combination of sigma values $(\sigma 1-\sigma 3)$ has a deviation of 0.5 . The reason the deviation in the sigma value is kept at 0.5 is that the image gets blurred as the sigma value rises above 0.5 , as can be seen in table 4.

From this table, it is clear that the concentrated pixels seem to appear brighter near the edges after the application of the Gaussian filter. But the sigma value and the convolution mask determine the extent of smoothing. When the sigma value is $0.5,1$ and 1.5 with the convolution mask 7, 7, the image gets blurred - with the white pixels at the centre and the dark pixels near the edges which removes all the noises. On the contrary, when the sigma value increases $($ sigma $=2,2.5$ and 3 and sigma $=$ $3.5,4$ and 4.5), the image gets extremely blurred, with difficulty in detecting edges. Therefore, in this table, a lower accuracy rate is noticed. It is also observed that if the sigma value and convolution mask increase simultaneously, the segmentation is inconclusive because the object gets invisible and cannot be used for further segmentation.

\subsection{Image quality estimation for the segmented output}

In line with the motivation of this work, it is necessary to denoise the image without loss of image quality. Therefore, this experiment is conducted to examine the quality of the image before and after applying the tri-channel method using the PSNR and MSE values presented in table 5.

The squared error between the original image and the reconstructed image is compared using the PSNR and the MSE, which share an inverse relationship with each other. Table 5 shows that the segmented image with the trichannel method has a high PSNR value and a low MSE value, indicative of the high quality of the image.

\subsection{Performance evaluation for the proposed segmentation method with the existing algorithm}

The proposed segmentation method is compared to the existing algorithm by calculating the probability of the given intensity belonging to the object pixel and the background pixel. Metrics like overlap-based metrics, the pair-counting-based method and distance measures [40] are used for the estimation of the proposed segmentation method with the existing method, described, respectively, in figures 6,7 and 8.

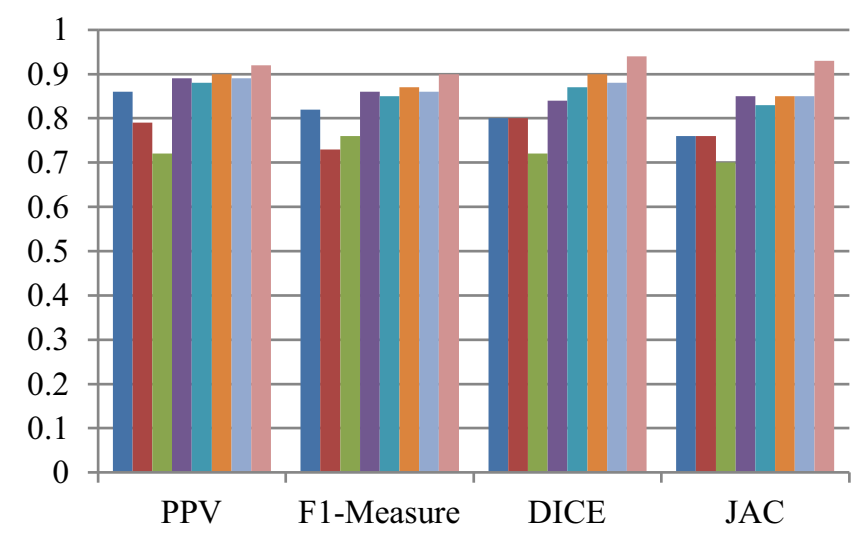

Figure 6. Overlap-based metrics for evaluating proposed method with existing algorithm.
- Region Growing [11]

- Morphological [10]

- Sparce Field Active Contour

[35]

NS and FCM clustering [8]

- INS and watershed [26]

NS Filtering and Level Set [29]

Neutrosophic and image

denoising [30]

Block Based Tri-Channel INS

and Sparse field Active 


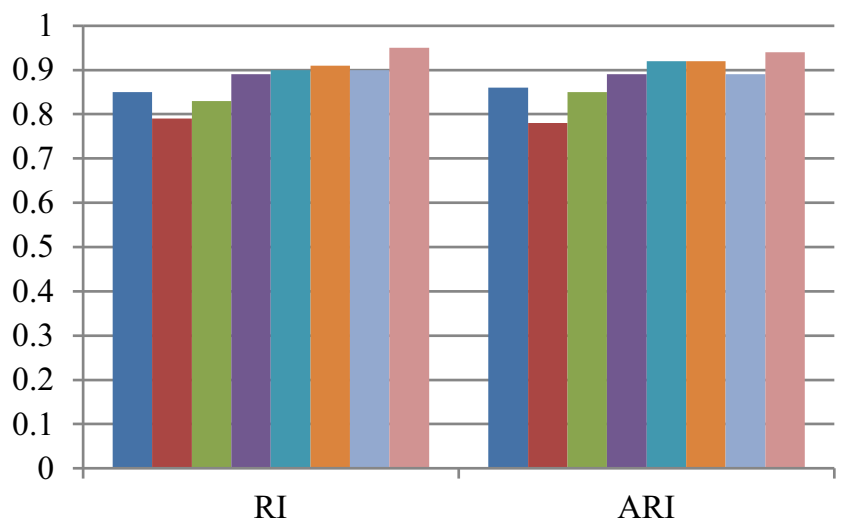
Region Growing [11]
- Morphological [10]
- Sparce Field Active Contour
[35]
NS and FCM clustering [8]
- INS and watershed [26]
NS Filtering and Level Set
[29]
Neutrosophic and image
denoising [30]
Block Based Tri-Channel
INS and Sparse field Active

Figure 7. Pair-counting-based method for evaluating existing algorithm with proposed approach.

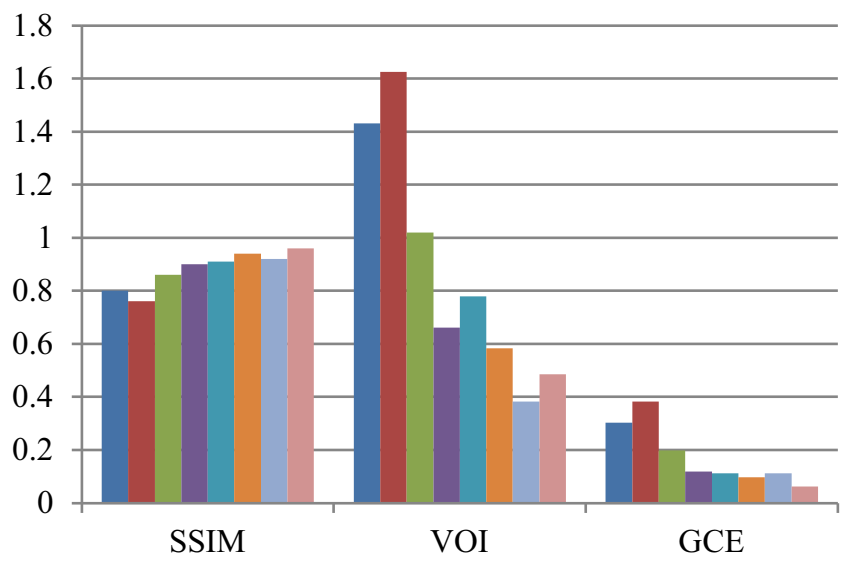

$$
\begin{aligned}
& \square \text { Region Growing [11] } \\
& \square \text { Morphological [10] } \\
& \text { Sparce Field Active Contour } \\
& {[35]} \\
& \square \text { NS and FCM clustering [8] } \\
& \text { INS and watershed [26] } \\
& \text { NS Filtering and Level Set } \\
& {[29]} \\
& \text { Neutrosophic and image } \\
& \text { denoising [30] } \\
& \text { Block Based Tri-Channel } \\
& \text { INS and Sparse field Active }
\end{aligned}
$$

Figure 8. Distance measures for evaluating existing algorithm with proposed approach.

In general, region growing and morphological segmentation are basic segmentation algorithms, and a comparison between the two demonstrates that the region growing algorithm performs better, as the experimental results in figures 6-8 reveal. Further, the region growing algorithm has been overtaken by the Sparse Field Active Contour. NS-based segmentation is being used in recent times for various applications involving image segmentation. The experiment clearly illustrates that the NS- and the INS-based segmentation achieve enhanced results. This motivated us to use the INS and Sparse Field Active Contour for developing the segmentation procedure. Finally, with the help of latest segmentation algorithms like the INS and the Sparse Field Active Contour, our proposed segmentation procedure has been experimented with it to show that it offers improved segmentation accuracy.

\section{Conclusion}

In this paper, a new segmentation procedure is developed using the NS and the Sparse Field Active Contour, which has a clear advantage in terms of blurred edges, noise reduction and improved segmentation accuracy. The proposed method is tested on natural image datasets. Four types of experiments were conducted. The first experiment discovers the block size and block category that provide overlapping blocks for the block size of $128 \times 8$ with good results. The second experiment finds the optimum value for the sigma and convolution mask. Superlative results are obtained for sigma values $0.5,1$ and 1.5 with the convolution mask 7, 7 for the overlapping block size of $128 \times 8$. The third experiment checks the competence of the proposed work in terms of noise tolerance and it is proved to be superior. Finally, the proposed method is compared with the existing method and it achieves a significant improvement in segmentation accuracy. In the future, this segmentation method can be implemented in real-time medical, biometrics and satellite image datasets.

\section{Reference}

[1] Rosenfeld A and Weinshall A 2011 Extracting foreground masks towards object recognition. In: Proceedings of the IEEE International Conference on Computer Vision (ICCV '11), pp. 1371-1378 
[2] Zhuang H, Low K S and Yau W Y 2012 Multichannel pulsecoupled-neural-network-based color image segmentation for object detection. IEEE Trans. Ind. Electron. 59(8): 3299-3308

[3] Behrens T, Rohr K and Stiehl H S 2003 Robust segmentation of tubular structures in 3-D medical images by parametric object detection and tracking. IEEE Trans. Syst. Man Cybern. Part B (Cybern.) 33(4): 554-561

[4] Wang Q, Chen F, Xu W and Yang M 2012 Object tracking via partial least squares analysis. IEEE Trans. Image Process. 21(10): 4454-4465

[5] Ozden M and Polat E 2007 A color image segmentation approach for content-based image retrieval. Pattern Recognit. 40(4): 1318-1325

[6] Borah B and Bhattacharyya D K 2005 Image retrieval by content using segmentation approach. In: Proceedings of the International Conference on Pattern Recognition and Machine Intelligence. Berlin, Heidelberg: Springer, pp. 551-556

[7] Levin A, Lischinski D and Weiss Y 2008 A closed-form solution to natural image matting. IEEE Trans. Pattern Anal. Mach. Intell. 30(2): 228-242

[8] Anter A M, Hassanien A E, ElSoud M A A and Tolba M F 2014 Neutrosophic sets and fuzzy C-means clustering for improving CT liver image segmentation. In: Proceedings of the Fifth International Conference on Innovations in BioInspired Computing and Applications (IBICA). Springer, Switzerland, pp. 193-203

[9] Chamorro-Martinez J, Sanchez D and Prados-Suarez B 2003 A fuzzy color image segmentation applied to robot vision. In: Advances in Soft Computing-Engineering, Design and Manufacturing, pp. 129-138

[10] Dougherty E R 1994 Morphological segmentation for textures and particles. Digit. Image Process. Methods 42: 43-102

[11] Fan J, Zeng G, Body M and Hacid M S 2005 Seeded region growing: an extensive and comparative study. Pattern Recognit. Lett. 26(8): 1139-1156

[12] Wang J, Ying Y, Guo Y and Peng Q 2006 Automatic foreground extraction of head shoulder images. In: Advances in Computer Graphics, pp. 385-396

[13] Stephanakis I M and Anastassopoulos G C 2006 Segmentation using adaptive thresholding of the image histogram according to the incremental rates of the segment likelihood functions. In: Proceedings of the 5th International Symposium on Communication Systems Networks and Digital Signal Processing, University of Patras, Greece, pp. 464-468

[14] Lee S W, Yang H S and Seo Y H 2013 Foreground extraction algorithm using depth information for image segmentation. In: Proceedings of Eighth IEEE International Conference on Broadband and Wireless Computing, Communication and Applications (BWCCA), pp. 581-584

[15] Qi C 2014 Maximum entropy for image segmentation based on an adaptive particle swarm optimization. Appl. Math. Inf. Sci. 8(6): 3129

[16] Yuan Y, Liu Y, Dai G, Zhang J and Chen Z 2014 Automatic foreground extraction based on difference of Gaussian. Sci. World J. https://doi.org/10.1155/2014/ 296074
[17] Qin L, Sheng B, Lin W, Wu W and Shen R 2015 GPUaccelerated video background subtraction using Gabor detector. J. Vis. Commun. Image Represent. 32: 1-9

[18] Yang B, Yu H and Hu R 2015 Unsupervised regions based segmentation using object discovery. J. Vis. Commun. Image Represent. 31: 125-137

[19] Yang Y, Guo L and Ye Y 2016 Robust natural image segmentation by using spatially constrained multivariate mixed Student's t-distribution and TV flow edge. J. Vis. Commun. Image Represent. 40: 178-196

[20] Pratondo A, Chui C K and Ong S H 2017 Integrating machine learning with region-based active contour models in medical image segmentation. J. Vis. Commun. Image Represent. 43: 1-9

[21] Akinlar C and Topal C 2017 ColorED: color edge and segment detection by edge drawing (ED). J. Vis. Commun. Image Represent. 44: 82-94

[22] Smarandache F 2005 A Unifying Field in Logics: Neutrosophic Logic. Neutrosophy, Neutrosophic Set, Neutrosophic Probability: Neutrsophic Logic. Neutrosophy, Neutrosophic Set, Neutrosophic Probability. Infinite Study. American Research Press, Rehoboth, NM

[23] Guo Y, Şengür A and Ye J 2014 A novel image thresholding algorithm based on neutrosophic similarity score. Measurement 58: $175-186$

[24] Wang H, Smarandache F, Sunderraman R and Zhang Y Q 2005 Interval neutrosophic sets and logic: theory and applications in computing. In: Neutrosophic Book Series, No. 5

[25] Wang H, Madiraju P, Zhang Y and Sunderraman R 2004 Interval neutrosophic sets. arXiv preprint math/0409113

[26] Zhang L and Zhang M 2015 Segmentation of blurry images based on interval neutrosophic set. J. Inf. Comput. Sci. 12(7): 2769-2777

[27] Mohan J, Guo Y, Krishnaveni V and Jeganathan K 2012. MRI denoising based on neutrosophic wiener filtering. In: Proceedings of the IEEE International Conference on Imaging Systems and Techniques (IST), pp. 327-331

[28] Zhang M, Zhang L and Cheng H D 2010 A neutrosophic approach to image segmentation based on watershed method. Signal Process. 90: 1510-1517

[29] Guo Y and Şengür A 2013 A novel image segmentation algorithm based on neutrosophic filtering and level set. Neutrosophic Sets Syst. 1(49): 46-49

[30] Guo Y, Cheng H D and Zhang Y 2009 A new neutrosophic approach to image denoising. New Math. Nat. Comput. 5(03): 653-662

[31] Cremers D, Rousson M and Deriche R 2007 A review of statistical approaches to level set segmentation: integrating color, texture, motion, and shape. Int. J. Comput. Vis. 72(2): 195-215

[32] Osher S and Fedkiw R 2003 Level Set Methods and Dynamic Implicit Surfaces. New York, NY: Cambridge University Press

[33] Sethian J A 1999 Level Set Methods and Fast Marching Methods. 2nd ed. New York, NY: Springer

[34] Whitaker R 1998 A level-set approach to 3D reconstruction from range data. Int. J. Comput. Vis. 29(3): 203-231

[35] Lankton S 2009 Sparse field methods. Technical Report, Georgia Institute of Technology 
[36] Lucas B C, Kazhdan M and Taylor R H 2012 Multi-object geodesic active contours (MOGAC): a parallel sparse-field algorithm for image segmentation. Department of Computer Science, Johns Hopkins University

[37] Murala S and Wu Q J 2015 Spherical symmetric 3D local ternary patterns for natural, texture and biomedical image indexing and retrieval. Neurocomputing 149: 1502-1514

[38] Zhang M 2010 Novel Approaches to Image Segmentation Based on Neutrosophic Logic. Utah State University

[39] Cheng H D, Wang J L and Shi X J 2004 Microcalcification detection using fuzzy logic and scale space approach. Pattern Recognit. 2(37): 363-375
[40] Taha A A and Hanbury A 2015 Metrics for evaluating 3D medical image segmentation: analysis, selection, and tool. BMC Med. Imaging 15(1): 29

[41] Pinki and Rajesh M 2016 Estimation of the image quality under different distortions. Int. J. Eng. Comput. Sci. 5(7): 17291-17296

[42] Rohit S 2013 Comparitive analysis of image segmentation techniques. Int. J. Adv. Res. Comput. Eng. Technol. 2(9): 2615-2619

[43] Xess M and Agnes S A 2014 Analysis of image segmentation methods based on performance evaluation parameters. Int. J. Comput. Eng. Res. 4(3): 68-75 